\title{
Nonequilibrium effects in the thermal switching of underdamped Josephson junctions
}

\author{
Juan José Mazo, ${ }^{1}$ Fernando Naranjo, ${ }^{1,2}$ and David Zueco ${ }^{1}$ \\ ${ }^{1}$ Departamento de Física de la Materia Condensada and Instituto de Ciencia de Materiales de Aragón, \\ CSIC-Universidad de Zaragoza, 50009 Zaragoza, Spain \\ ${ }^{2}$ Universidad Pedagógica y Tecnológica de Colombia, Tunja, Colombia
}

(Received 27 July 2010; published 3 September 2010)

\begin{abstract}
We study the thermal escape problem in the low damping limit. We find that finiteness of the barrier is crucial for explaining the thermal activation results. In this regime, low barrier nonequilibrium corrections to the usual theories become necessary. We propose a simple theoretical extension accounting for these nonequilibrium processes which agrees numerical results. We apply our theory to the understanding of switching current curves in underdamped Josephson junctions.
\end{abstract}

DOI: 10.1103/PhysRevB.82.094505

PACS number(s): 74.50. $+\mathrm{r}, 05.40 .-\mathrm{a}$

\section{INTRODUCTION}

In 1940, Kramers derived his famous formulas describing rates in chemical reactions. ${ }^{1}$ The theoretical framework for his calculation was the escape of a Brownian particle over a potential barrier. Far from being a particular case, noiseactivated escape is applicable in a wide number of problems in science, going from biology to quantum information processing. ${ }^{2}$ Due to the many fields involved, intense activity emerged in the subject proposing better theories for this, nowadays, old problem. ${ }^{2-4}$

In particular, studies on thermal switching in Josephson junctions (JJs) benefits from this effort. ${ }^{5-9}$ Experimental results are affected by thermal fluctuations and measurements in the laboratory allow to predict junctions parameters by fitting the switching with available expressions. Also, some fundamental issues as the quantum-classical transition have been addressed by means of rates measurements. ${ }^{7,8,10}$ It is clear, that such a measurements need to be compared with appropriate theoretical results. Needless to say the exact formula does not exist and many theories are available in the literature, which starting from the Kramers seminal work, cover different set of parameters. ${ }^{3,4}$

In a recent numerical work, ${ }^{11}$ for very low values of the damping parameter, it has been found a significant deviation of the JJ switching current from the expected result. Here we present a theory that is able to give account for the observed deviation. Moreover, we predict that this effect will appear in any biased system where the damping over force ramp ratio is not large. In such a case, the usual theories are not suitable, and, as we show, it is needed to include nonequilibrium effects and finite barrier correction in a full description of the problem.

To be definite, the dynamics for the phase difference in the junction is usually described by the, so-called, resistively and capacitively shunted junction model, which is equivalent to the more general problem of a Brownian particle in a metastable potential,

$$
m \ddot{x}+m \gamma \dot{x}=-\frac{d V}{d x}+\xi(t),
$$

where the potential $V(x)=V_{0}(1-\cos x)-I x$ and $\xi(t)$ is the stochastic force describing the thermal fluctuations.
Here we consider white thermal noise, $\langle\xi(t)\rangle=0$ and $\left\langle\xi(t) \xi\left(t^{\prime}\right)\right\rangle=2 m \gamma k_{B} T \delta\left(t-t^{\prime}\right)$.

For moderate to low values of the damping parameter, there exists a temperature-dependent critical current (force) for the system to switch from a superconducting or locked state $(\langle\dot{x}\rangle=0)$ to a resistive or running one $(m \gamma\langle\dot{x}\rangle=I)$. Such a situation corresponds to the problem of escape from a metastable well. In switching current experiments many currentvoltage (force-velocity) curves are performed to obtain the switching current probability distribution function, $P(I)$. The measured $P(I)$ can be directly related to the thermal activation rate $^{6}$ and experimental results can be understood in terms of such parameter.

For very weak damping, the Kramers result for the activation rate is $r_{K L D}=\left(\gamma J_{b} / k_{B} T\right)\left(\omega_{a} / 2 \pi\right) e^{-\Delta U / k_{B} T}$, where $\omega_{a}$ is the frequency at the bottom of the well and $J_{b}$ the value of the action at the top of the barrier. There we recognize the transition-state-theory result multiplied by a prefactor valid in the very low damping regime. For our system, the action at the barrier $J_{b}$ is usually approached by the cubic potential result $J_{b}=7.2 \Delta U / \omega_{a}$. Then

$$
r_{K L D} \simeq \frac{7.2 \gamma}{2 \pi} \frac{\Delta U}{k_{B} T} e^{-\Delta U / k_{B} T}
$$

This equation shows that the rate scales linearly with the damping and depends only on the barrier height over thermal energy factor, $\Delta U / k_{B} T$. This expression is only valid in the low damping and infinite barrier limit $\left(\gamma J_{b} / k_{B} T \ll 1\right.$ and $\left.\Delta U / k_{B} T \gg 1\right)$.

Many theories have extended the Kramers result to the moderate-to-small damping regime ${ }^{3,12-14}$ following the infinite barrier approximation. Given its simplicity, the result of Büttiker-Harris-Landuer (BHL) (Ref. 12) has been usually applied in the JJ literature. Finite barrier corrections have been studied in Refs. 15 and 16. More recently, Drozdov and Hayashi (DH) proposed a new theory which is not perturbative in the barrier height. ${ }^{17,18}$

We are interested in the dynamics of the system in the low damping limit. In this limit, the coupling to the bath is very weak and the time to reach thermal equilibrium very long $(\sim 1 / \gamma)$. This fact has important consequences. For biased systems, escape occurs at very low values of the $\Delta U / k_{B} T$ 

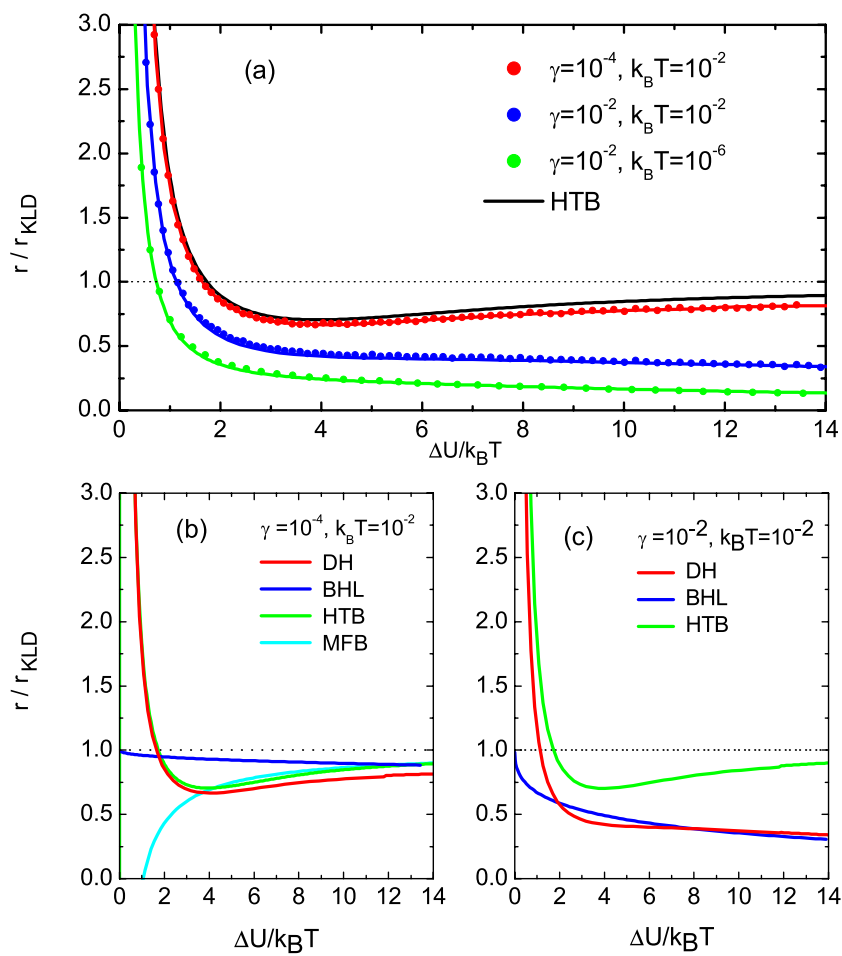

FIG. 1. (Color online) Escape rate divided by the Kramers low damping result, Eq. (2), as a function of $\Delta U / k_{B} T$. (a) Dots are numerical results and the corresponding color solid lines are the theoretical prediction by DH theory (Ref. 18). The black line stands for the vanishing damping rate formula, Eq. (3). (b) and (c) compare different theoretical results.

ratio and junctions may escape before thermal equilibrium is established and thus nonequilibrium effects dominate the process. In order to study such effects we need first to know the importance of finite barrier effects in particle activation problem at low damping and take into account the average energy of junctions before each switching event.

\section{ESCAPE AT SMALL BARRIER}

We will show here that small barrier effects are very important in the low damping case, the convergence to the infinite barrier result is very slow and the DH theory reproduces the numerical results at any barrier.

We have numerically integrated the Langevin Eq. (1) of the system for different values of damping and barrier height. ${ }^{19}$ In our simulations, we have computed the mean time for the system to first reach the potential barrier. For low values of the damping, such mean time (the firstpassage-time problem) corresponds to the inverse of the escape rate. According to theory, simulations are started with particles placed in the metastable potential well and zero velocity. Some issues about the initial conditions problem will be addressed below. At any point, the numerical result is obtained from $10^{4}$ escaping events. We show results for $V_{0}=0.155, m=0.35$ and different values of $I$, damping and temperature. The results are summarized in Figs. 1 and 2 where we plot the activation rate as a function of barrier and
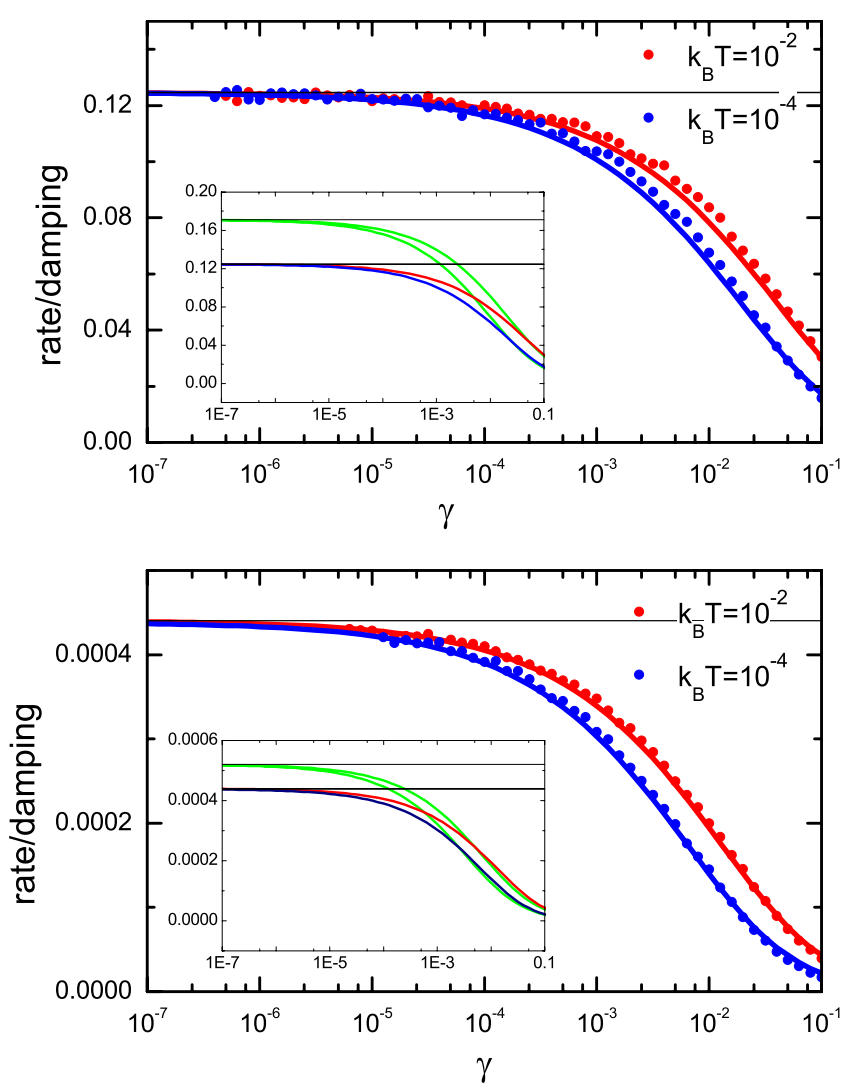

FIG. 2. (Color online) Damping dependence for the escape rates. The points are numerical results and the corresponding solid lines account for DH theory (Ref. 18). The horizontal black line is the vanishing damping limit Eq. (3). Top: curves for $\Delta U / k_{B} T=3$. Bottom: $\Delta U / k_{B} T=10$. The inset compares $\mathrm{DH}$ to $\mathrm{BHL}$ (green lines) results.

damping, respectively, and compare to some existing theories.

Figure 1 shows the rate dependence on the barrier for different values of damping and temperature. In order to see deviations from the Kramers low damping result, we divide the obtained rates by Eq. (2). We recall that Eq. (2) is obtained assuming weak damping and high barrier. For comparison, we also plot the exact result for arbitrary barrier in the limit of vanishing damping, ${ }^{3} r(\gamma \rightarrow 0)=r_{H T B}$ with

$$
r_{H T B}=\gamma k_{B} T\left[\int_{0}^{J_{b}} d J e^{-\beta E(J)} \int_{J}^{J_{b}} d J^{\prime} \frac{\omega\left(J^{\prime}\right)}{2 \pi} \frac{e^{\beta E\left(J^{\prime}\right)}}{J^{\prime}}\right]^{-1}
$$

with $E(J)$ is the energy as a function of the action $J$ and $\omega(J)=2 \pi \partial_{J} E(J), J_{b}$ is the action at the barrier and $\beta=1 / k_{B} T$.

Remarkably, the approach between both results is slow, meaning that the high barrier approximation is accurate only at very high barrier values indeed [Fig. 1(a)]. For instance, $r_{H T B} / r_{K L D}=0.72$ for $\Delta U / k_{B} T=5$ and 0.85 for $\Delta U / k_{B} T=10$. As a consequence, all theories which try to extend the Kramers result to the moderate-to-small damping region ${ }^{3,12-14}$ also fail at low damping values unless very large barriers are considered. 
We know about two main attempts to include finite barrier effects in this limit: the first one is due to MFB (Mel'nikov Finite Barrier) (Ref. 15) and fails at small barriers, see Fig. 1(b). The second one was proposed by DH for moderate-tosmall damping and arbitrary barrier. ${ }^{17,18}$ As it can be seen in Figs. 1 and 2, the DH theory recovers the vanishing damping limit and explain our numerical results in the whole small damping region. In Figs. 1(b) and 1(c), we show BHL result. ${ }^{20}$ Other extensions of the Kramers low damping result to the moderate-to-low damping regime give a result quite similar to BHL.

In Fig. 2, we plot the rate damping dependence for two different barriers. Apart from the agreement to the $\mathrm{DH}$ theory, we check the convergence to the very weak limit given by Eq. (3) [cf. horizontal line in both figures]. In the inset, we also show results for the BHL. We see that the finite barrier corrections become less important by increasing the damping.

To complete our discussion, we notice that by decreasing the damping the curves at different temperatures become the same, that is the rate depends only on the ratio $\Delta U / k_{B} T$. This can be understood by noticing that most of the contribution in the integrals in Eq. (3) comes from the bottom of the potential. If the action inside the well is approximated by the corresponding one for an harmonic potential with the same bottom frequency, $E=\left(\omega_{0} / 2 \pi\right) J$, with $\omega_{0}=\partial_{x}^{2} V\left(x_{\text {min }}\right)$, the rate Eq. (3) can be written as,

$$
r_{H A}=\gamma\left[\int_{0}^{\Delta U / k_{B} T} d x e^{-x} \int_{x}^{\Delta U / k_{B} T} d y \frac{e^{y}}{y}\right]^{-1},
$$

which clearly depends only on the ratio $\Delta U / k_{B} T$. Let us emphasize that, besides its simplicity, the above equation is an excellent approximation to Eq. (3). In fact, plotting both Eqs. (3) and (4) in Fig. 1, they cannot be distinguished one from the other.

\section{INITIAL CONDITIONS DEPENDENCE}

We address now the influence of the initial condition for the energy on the escape rate results. Thermal escape at low damping is an energy diffusion problem. Escape occurs as soon as thermal fluctuations provide a particle energy enough to overcome the barrier. This time depends on the value of the particle initial energy.

Up to now, to compare our simulations to theory we assumed that the particle starts at the bottom of the metastable well with zero velocity. From the experimental point of view, this assumption may fail. Thermal fluctuations not only provide energy enough to surmount the barrier but also kinetic energy at the bottom. In order to study the importance of this issue, in Fig. 3 we plot the rates with two initial conditions, $v= \pm \sqrt{k_{B} T / m}$ and compare to the one with zero velocity. As expected, we see that for small barriers initial kinetic energy speed up the escape times.

When particles are placed with zero velocity at the bottom of the well, the activation time is $r^{-1}$. However, if particles have extra initial energy $E_{i n}$ the escape time is given by $r^{-1}-\tau$, where $\tau$ is the activation time up to this extra energy,

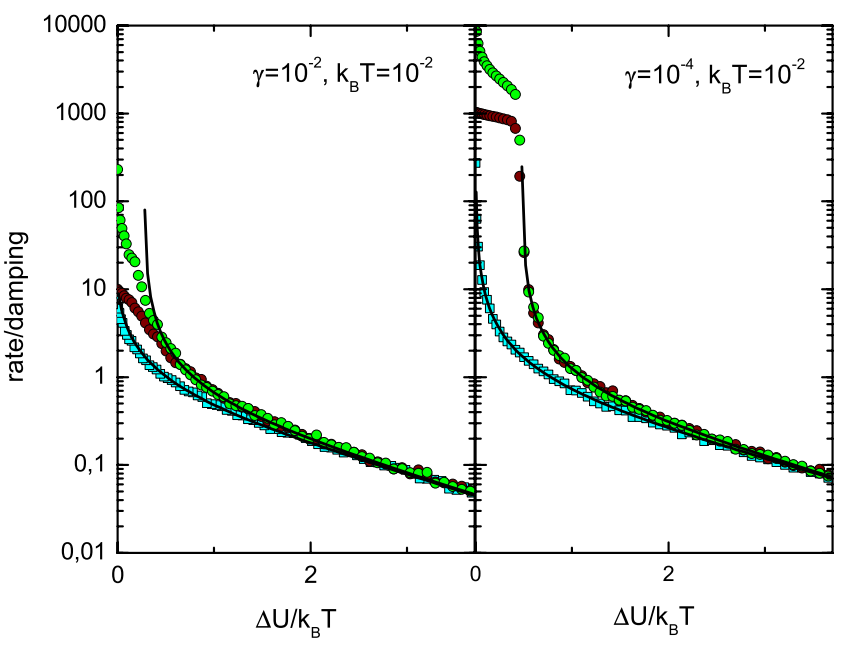

FIG. 3. (Color online) Activation rate vs barrier for three initial conditions: particles are at the bottom of the metastable well with zero velocity (blue squares), $v=\sqrt{k_{B} T / m}$ (green light points), or $v=-\sqrt{k_{B} T / m}$ (brown dark points). Lines stand for theoretical predictions of Eq. (5).

which can be computed at low damping from Eq. (4) replacing $\Delta U$ by $E_{i n}$. Putting all together, we generalize the rate formulas as,

$$
r_{i n}=\frac{1}{r^{-1}-\tau} .
$$

This equation shows that as soon as $\tau\left(E_{i n}\right) \sim r^{-1}$, the initial conditions problem affect the escape rates. In Fig. 3, where $E_{i n}=k_{B} T / 2$, this correction becomes important for $\Delta U / k_{B} T \lesssim 2$. If $\Delta U \leq E_{i n}$, the passage time is almost a deterministic process which depends on the initial position and velocity of the system. Figure 3 illustrates this effect and confirms our theoretical prediction.

\section{SWITCHING CURRENT}

In a typical $\mathrm{JJ}$ experiment, the probability distribution function of the junction switching current $P(I)$ is measured performing many current-voltage curves where current is continuously increased at a given rate. From these results, the mean switching current $I_{\mathrm{sw}}$ and its standard deviation can be trivially computed. Such $P(I)$ can be easily related to the escape rates $r(I)$ as ${ }^{6}$

$$
P(I)=r(I)\left(\frac{d I}{d t}\right)^{-1}\left[1-\int_{0}^{I} P(u) d u\right] .
$$

Alike, escape rates can be computed from measured $P(I)$.

Figure 4(a) shows our numerical results for the average switching current and compares them to theoretical predictions. We integrate Eq. (1) for an ensemble of thermalized junctions. Current is increased at a given ramp and switching events are recorded. As expected BHL-based predictions fail in the very low damping regime. However, surprisingly, also DH is unable to explain our numerical results, which lie in between both theories. This is due to the competition be- 

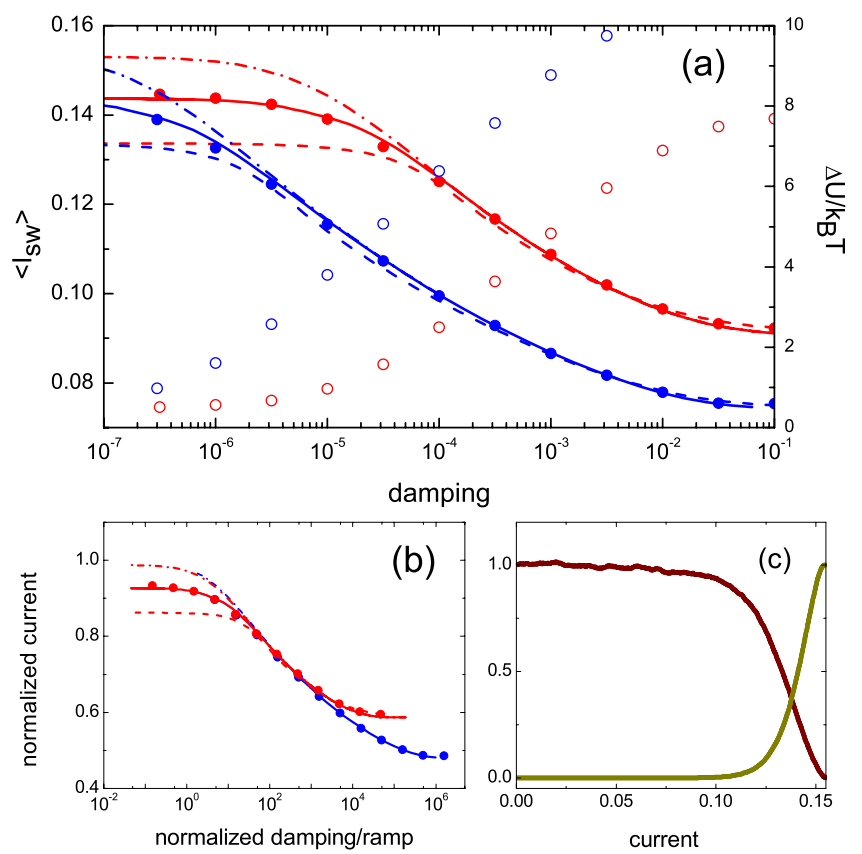

FIG. 4. (Color online) (a) Average switching current at different values of the damping for $T=0.01$ and current ramp $\dot{I}=3.33 \times 10^{-7}$ (red) and $\dot{I}=10^{-8}$ (blue). Solid symbols are for numerical simulations, dashed lines for predictions based on BHL theory, dotted lines for DH theory, and solid lines for our theory, Eq. (5) with $E_{i n}=k_{B} T / 2$. We also plot (right axis) the value of the barrier at the mean switching current. (b) Normalized current versus normalized damping over ramp ratio (Ref. 21). (c) Mean energy divided by $k_{B} T$ of particles in the well (brown) and fraction of particles which have escaped (green) as a function of the applied current $\left(T=0.01, \gamma=10^{-5}\right.$, and $\left.\dot{I}=3.33 \times 10^{-7}\right)$.

tween the equilibrium time of the system, given by $\gamma^{-1}$, and the time order for the change in the current, given by the inverse of the current ramp. Thus, switching in the very low damping regime is a nonequilibrium process. The coupling to the external bath is so weak that other junctions are not able to reach the thermal energy before switching. Thus, junctions escape in an evaporative cooling way where more energetic junctions switch first and the ensemble is effectively cooled. This picture is confirmed in Fig. 4(c) where for a given damping we show the mean energy for the trapped junctions as a function of the current and the fraction of particles which have switched.

We also see in Fig. 4(c) that particles escape with an initial energy which goes from $E_{i n}=K_{B} T$ to zero when current is increased. The simplest way to introduce this fact in the theory is to assume an average value of $E_{i n}=K_{B} T / 2$ and use our Eq. (5). Figure 4(a) shows that in this way, we are able to reproduce quite accurately the numerical results. This correction turns out to be important when the average barrier at the switching current is on the order of the thermal energy. See that in the figure, it is also plotted the value of the barrier at the mean switching current (open symbols). Finally, using Eqs. (4)-(6) it can be seen that in this region of the parameter space, the results depend on the $\gamma / \dot{I}$ ratio, as confirmed in Fig. 4(b). Therefore, our theory allows to estimate the values for $\gamma / \dot{I}$ where nonequilibrium corrections are necessary.

\section{OUTLOOK}

We have chosen to present our results in the framework of JJ devices. However, given the wide interest of thermal activation in many scientific areas, our results can be applied to many other systems coupled weakly to the environment. ${ }^{22,23}$ Suggested by our findings an important issue to study, it is the influence of the observed competition between two different time scales on results for biased systems at higher values of the damping and thus transfer our theoretical scheme from the energy-diffusion regime to the phasediffusion one. This is the typical case for many of the current friction $^{24}$ and biological-physics experiments. ${ }^{25}$

\section{ACKNOWLEDGMENTS}

We thank F. Falo and L. M. Floría for discussions and critical reading of the manuscript. This work was supported by Spain MICINN under Project No. FIS2008-01240, cofinanced by FEDER funds.
${ }^{1}$ H. Kramers, Physica (Amsterdam) 7, 284 (1940).

${ }^{2}$ E. Pollak and P. Talkner, Chaos 15, 026116 (2005).

${ }^{3}$ P. Hänggi, P. Talkner, and M. Borkovec, Rev. Mod. Phys. 62, 251 (1990).

${ }^{4}$ V. I. Mel'nikov, Phys. Rep. 209, 1 (1991).

${ }^{5}$ V. Ambegaokar and B. I. Halperin, Phys. Rev. Lett. 22, 1364 (1969); M. J. Stephen, Phys. Rev. 186, 393 (1969); J. Kurkijärvi, Phys. Rev. B 6, 832 (1972).

${ }^{6}$ T. A. Fulton and L. N. Dunkleberger, Phys. Rev. B 9, 4760 (1974).

${ }^{7}$ S. Washburn, R. A. Webb, R. F. Voss, and S. M. Faris, Phys. Rev. Lett. 54, 2712 (1985).

${ }^{8}$ M. H. Devoret, J. M. Martinis, and J. Clarke, Phys. Rev. Lett. 55, 1908 (1985); J. M. Martinis, M. H. Devoret, and J. Clarke,
Phys. Rev. B 35, 4682 (1987).

${ }^{9}$ P. Silvestrini, O. Liengme, and K. E. Gray, Phys. Rev. B 37, 1525 (1988).

${ }^{10}$ A. Wallraff, A. Lukashenko, J. Lisenfeld, A. Kemp, M. V. Fistul, Y. Koval, and A. V. Ustinov, Nature (London) 425, 155 (2003).

${ }^{11}$ J. J. Mazo, F. Naranjo, and K. Segall, Phys. Rev. B 78, 174510 (2008).

${ }^{12}$ M. Büttiker, E. P. Harris, and R. Landauer, Phys. Rev. B 28, 1268 (1983).

${ }^{13}$ H. Grabert, Phys. Rev. Lett. 61, 1683 (1988); E. Pollak, H. Grabert, and P. Hänggi, J. Chem. Phys. 91, 4073 (1989).

${ }^{14}$ V. I. Mel'nikov and S. Meshkov, J. Chem. Phys. 85, 1018 (1986).

${ }^{15}$ V. I. Mel'nikov, Phys. Rev. E 48, 3271 (1993). 
${ }^{16}$ R. Ferrando, R. Spadacini, G. E. Tommei, and V. I. Melnikov, Phys. Rev. E 51, R1645 (1995).

${ }^{17}$ A. N. Drozdov and S. Hayashi, Phys. Rev. E 60, 3804 (1999).

${ }^{18} r_{\mathrm{DH}}=A\left(r_{H T B} / \Gamma_{T S T}\right) \times \Gamma_{T S T}$ where $r_{H T B}$ is given by Eq. (3), $\Gamma_{T S T}$ is the transition-state-theory rate, $\Gamma_{T S T}$ $=\left\{\sqrt{2 \pi \beta m} \int_{-\infty}^{0} d x e^{-\beta V(x)}\right\}^{-1}$ and $A(y)$ is the interpolation function given by Mel'nikov and Meshkov (Ref. 14): $A(y)$ $=\exp \left[\frac{1}{\pi} \int_{0}^{\infty} d x \ln \left\{1-\exp \left[-y\left(x^{2}+\frac{1}{4}\right)\right]\right\} /\left(x^{2}+\frac{1}{4}\right)\right]$.

${ }^{19}$ The equations were numerically integrated using the Stochastic Runge Kutta algorithm $(3 \mathrm{o} 4 \mathrm{~s} 2 \mathrm{~g})$ with time step 0.01 .

${ }^{20}$ For the correction factor $\alpha$ introduced in the BHL theory (Ref. 12), we use the standard value $\alpha=1$.

${ }^{21}$ To normalize Eq. (1), we divide it by $V_{0}$ and time by $\omega^{-1}$ $=\sqrt{m / V_{0}}$. Then, the adimensionalized parameters are $\tilde{\gamma}=\gamma / \omega$
$=1.50 \gamma, \tilde{T}=k_{B} T / V_{0}=6.45 k_{B} T$, and $\tilde{\dot{I}}=\dot{I} /\left(\omega V_{0}\right)=9.70 \dot{I}$.

${ }^{22}$ D. J. McCarthy and W. T. Coffey, J. Phys.: Condens. Matter 11, 10531 (1999); J. L. García-Palacios and F. J. Lázaro, Phys. Rev. B 58, 14937 (1998).

${ }^{23}$ J. A. Pelesko and D. H. Bernstein, Modeling MEMS and NEMS (Chapman and Hall/CRC, Boca Raton, 2003).

${ }^{24}$ Y. Sang, M. Dubé, and M. Grant, Phys. Rev. Lett. 87, 174301 (2001); I. Barel, M. Urbakh, L. Jansen, and A. Schirmeisen, ibid. 104, 066104 (2010); O. K. Dudko, A. E. Filippov, J. Klafter, and M. Urbakh, Proc. Natl. Acad. Sci. U.S.A. 100, 11378 (2003).

${ }^{25}$ C. Hyeon and D. Thirumalai, Proc. Natl. Acad. Sci. U.S.A. 100, 10249 (2003); O. K. Dudko, ibid. 106, 8795 (2009). 